\title{
A!
}

This is an electronic reprint of the original article.

This reprint may differ from the original in pagination and typographic detail.

Singh, Nishant K.; Käpylä, Maarit J.; Brandenburg, Axel; Käpylä, Petri J.; Lagg, Andreas; Virtanen, Ilpo

\section{Bihelical Spectrum of Solar Magnetic Helicity and Its Evolution}

\section{Published in:}

The Astrophysical Journal

DOI:

10.3847/1538-4357/aad0f2

Published: 20/08/2018

\section{Document Version}

Publisher's PDF, also known as Version of record

Please cite the original version:

Singh, N. K., Käpylä, M. J., Brandenburg, A., Käpylä, P. J., Lagg, A., \& Virtanen, I. (2018). Bihelical Spectrum of Solar Magnetic Helicity and Its Evolution. The Astrophysical Journal, 863(2), 1-11. [182].

https://doi.org/10.3847/1538-4357/aad0f2

This material is protected by copyright and other intellectual property rights, and duplication or sale of all or part of any of the repository collections is not permitted, except that material may be duplicated by you for your research use or educational purposes in electronic or print form. You must obtain permission for any other use. Electronic or print copies may not be offered, whether for sale or otherwise to anyone who is not an authorised user. 


\title{
Bihelical Spectrum of Solar Magnetic Helicity and Its Evolution
}

\author{
Nishant K. Singh ${ }^{1}$ (D) , Maarit J. Käpylä ${ }^{1,2}$ (D), Axel Brandenburg ${ }^{3,4,5,6}$ (iD), Petri J. Käpylä ${ }^{1,2,7}$, Andreas Lagg ${ }^{1}$, and Ilpo Virtanen ${ }^{8}$ (D) \\ ${ }^{1}$ Max Planck Institute for Solar System Research, Justus-von-Liebig-Weg 3, D-37077 Göttingen, Germany \\ ${ }^{2}$ ReSoLVE Centre of Excellence, Department of Computer Science, Aalto University, PO Box 15400, FI-00076 Aalto, Finland \\ ${ }^{3}$ NORDITA, KTH Royal Institute of Technology and Stockholm University, Roslagstullsbacken 23, SE-10691 Stockholm, Sweden \\ ${ }^{4}$ Department of Astronomy, AlbaNova University Center, Stockholm University, SE-10691 Stockholm, Sweden \\ 5 JILA and Department of Astrophysical and Planetary Sciences, Box 440, University of Colorado, Boulder, CO 80303, USA \\ ${ }^{6}$ Laboratory for Atmospheric and Space Physics, 3665 Discovery Drive, Boulder, CO 80303, USA \\ ${ }^{7}$ Leibniz Institute for Astrophysics Potsdam, An der Sternwarte 16, D-14482 Potsdam, Germany \\ ${ }^{8}$ ReSoLVE Centre of Excellence, Space Climate research unit, University of Oulu, P.O.Box 3000 FI-90014 Oulu, Finland \\ Received 2018 April 13; revised 2018 June 25; accepted 2018 July 1; published 2018 August 22
}

\begin{abstract}
Using a recently developed two-scale formalism to determine the magnetic helicity spectrum, we analyze synoptic vector magnetograms built with data from the Vector Spectromagnetograph instrument on the Synoptic Optical Long-term Investigations of the Sun telescope during 2010 January-2016 July. In contrast to an earlier study using only three Carrington rotations (CRs), our analysis includes 74 synoptic CR maps. We recover here bihelical spectra at different phases of solar cycle 24, where the net magnetic helicity in the majority of the data is consistent with a large-scale dynamo with helical turbulence operating in the Sun. More than $20 \%$ of the analyzed maps, however, show violations of the expected sign rule.
\end{abstract}

Key words: dynamo - magnetohydrodynamics (MHD) - Sun: magnetic fields - turbulence

\section{Introduction}

Magnetic helicity is a topological invariant of ideal magnetohydrodynamics (MHD). It is a measure of complexity or internal twist of the magnetic field structure and has a geometrical interpretation in terms of linkage of magnetic field lines (Moffatt 1969; Berger \& Field 1984; Arnold \& Khesin 1992; Pevtsov et al. 2014). Moreover, it is expected to be nearly conserved even in nonideal MHD systems with large magnetic Reynolds number $R_{\mathrm{m}}$. This conservation law has been recently tested in a solar context involving magnetic reconnection (Pariat et al. 2015). Magnetic helicity thus plays a crucial role in the evolution of magnetic fields, and it can be an effective tracer of the underlying mechanism responsible for the generation of magnetic fields (Brandenburg \& Subramanian 2005).

There has been considerable interest in monitoring the magnetic helicity of active regions (ARs), as this characterizes the complexity of the ARs involved and is therefore often linked to its "eruptibility," causing solar flares and coronal mass ejection; see, e.g., Nindos et al. (2003), Valori et al. (2016), and also Pariat et al. (2017), who suggest a better "eruptivity proxy" involving magnetic helicity. Instead of being merely elements of the entire solar magnetic structure, the ARs, and the magnetic helicity they carry, play an important role for the global solar dynamo. The dynamogenerated large-scale field, by a mechanism still under some debate, feeds the localized magnetic concentrations, leading to the formation of ARs and sunspots. The thereby-formed ARs can contribute to the migration of the small-scale magnetic helicity, which is created as a by-product of the helically driven large-scale dynamo (LSD), away from the dynamo region, to prevent the quenching of the LSD (see, e.g., Brandenburg et al. 2003; Longcope \& Pevtsov 2003; Brandenburg \& Subramanian 2005, and references therein).

Although the origin of solar magnetism is yet to be fully understood, it is commonly thought that the global cyclic magnetic field of the Sun is generated and maintained by a turbulent dynamo (Vainshtein \& Zeldovich 1972; Moffatt 1978; Krause \& Rädler 1980; Ossendrijver 2003; Solanki et al. 2006; Charbonneau 2010). The solar dynamo is expected to involve an $\alpha$ effect, which is a measure of the helicity of turbulence in the convection zone caused by strong stratification and rotation (Krause \& Rädler 1980). Numerical simulations have shown that a significant $\alpha$ effect is indeed produced under these conditions in convective turbulence (e.g., Ossendrijver et al. 2002; Käpylä et al. 2009; Warnecke et al. 2018). It is known that the $\alpha$ effect produces a bihelical magnetic field where the magnetic helicities at large and small scales have opposite signs, and thus there is no net production of magnetic helicity in the process (Seehafer 1996; Ji 1999; Blackman \& Brandenburg 2003).

In the mean-field framework, the quantities, say, magnetic fields, $\boldsymbol{B}$, are expressed as a sum of mean $(\overline{\boldsymbol{B}})$ and fluctuating (b) components, i.e., $\boldsymbol{B}=\overline{\boldsymbol{B}}+\boldsymbol{b}$, giving two contributions for the magnetic helicity: $\mathcal{H}_{\mathrm{M}}=\langle\boldsymbol{A} \cdot \boldsymbol{B}\rangle=\langle\overline{\boldsymbol{A}} \cdot \overline{\boldsymbol{B}}\rangle+\langle\boldsymbol{a} \cdot \boldsymbol{b}\rangle$, with $\boldsymbol{A}$ being the vector potential defined from $\boldsymbol{\nabla} \times \boldsymbol{A}=\boldsymbol{B}$ (Krause \& Rädler 1980; Brandenburg \& Subramanian 2005). Angle brackets \langle\rangle denote the volume averages, while the overbars indicate ensemble or longitudinal averages, satisfying the Reynolds rules, for example, $\boldsymbol{a}=0$ and $\langle\overline{\boldsymbol{A}} \cdot \boldsymbol{b}\rangle=0$ (Krause \& Rädler 1980). This now helps us to summarize the expected hemispheric sign rule (HSR) of solar magnetic helicity, where the $\alpha$ effect changes sign across the equator: the local (global) magnetic helicity is expected to be negative (positive) in the northern hemisphere, and vice versa in the southern hemisphere, as shown schematically in Figure 1. The concept of scales is important in the present context where typical extents of even the largest ARs or the sunspots are considered small, whereas scales of the order of the solar radius are termed as large.

The HSR is confirmed in a number of earlier works reporting measurements of local as well as global magnetic net helicity at different phases of the solar cycle (SC), using different techniques that often involve determining the vector potential under a 


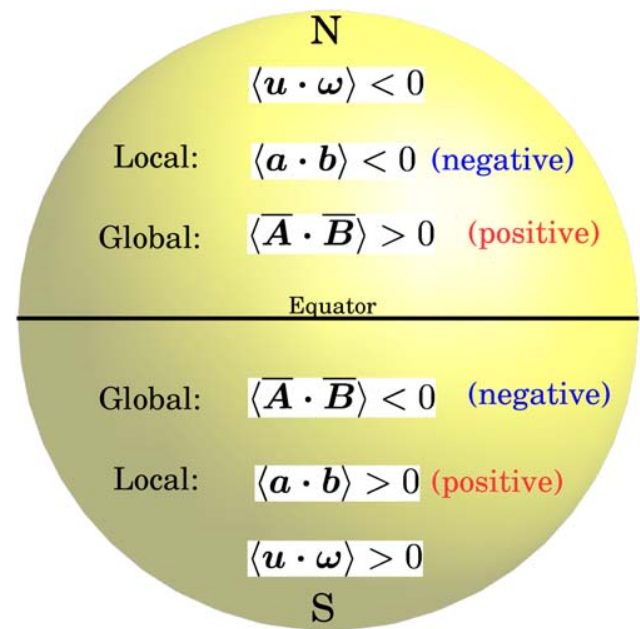

Figure 1. Schematic diagram showing the HSR of solar magnetic helicity as expected from an $\alpha$-driven turbulent dynamo.

suitable gauge choice (see, e.g., the method of Brandenburg et al. 2003). Using this method on data from the Michelson Doppler Imager (MDI) on board the Solar and Heliospheric Observatory $(\mathrm{SOHO})$ for SC 24 and Synoptic Optical Long-term Investigations of the Sun (SOLIS) data for SC 23, Pipin \& Pevtsov (2014) found that the global magnetic helicity was indeed positive (negative) in the northern (southern) hemisphere during SC 23 and SC 24, thus obeying the HSR as shown in Figure 1. The importance of such measurements in the solar context was discussed much earlier (Seehafer 1990), and many subsequent works, focusing mainly on the ARs contributing thus to the local measurements of the helicity, found that it is mostly negative (positive) in the north (south)_exactly according to the expected sign rule (see, e.g., Pevtsov et al. 1995; Bao et al. 1999; Liu et al. 2014a). Liu et al. (2014b) made an attempt to test the HSR using HMI data and found that it was obeyed by nearly $75 \%$ of all the ARs that they studied. Zhang et al. (2012) showed that the current helicity associated with ARs traces the magnetic helicity of the large scale magnetic field.

The dependence of magnetic helicity on the phase of the SC has been explored to some extent. Brandenburg et al. (2003) reported that the global magnetic helicity was negative before the solar maximum and turned positive afterward, i.e., they found evidence of a "wrong" sign during the rising phase of the cycle. Similar results were obtained by Pipin \& Pevtsov (2014) for SC 23 and SC 24 from MDI and SOLIS data. Many studies also utilize the current helicity, $\mathcal{H}_{\mathrm{C}}=\langle\boldsymbol{J} \cdot \boldsymbol{B}\rangle$, where $\boldsymbol{J}=$ $\mu_{0}^{-1} \nabla \times \boldsymbol{B}$ is the current density, as a proxy for the magnetic helicity and argue that these quantities can be used interchangeably (e.g., Zhang et al. 2010). This holds strictly only for magnetic and current helicity spectra, and only under isotropic conditions, which are in general not met under solar conditions. The study of Zhang et al. (2010) showed that the current helicity follows the equatorward propagation of magnetic dynamo wave traced through the sunspots in the photosphere. While much of their analysis confirms the HSR, they do also notice wrong signs of the helicity, mostly at the beginning and end of the cycle, and interpreted this as due to penetration of the activity wave into the other hemisphere. Also employing the current helicity method on SOLIS data, Gosain et al. (2013), however, found no such violations during the early phase of SC 24.
It is only recently that, instead of computing net magnetic helicities over a given domain, methods for computing magnetic helicity distribution over different spatial scales (spectrum) were developed. These were first applied to local patches of photospheric magnetic field measurements for a few ARs (Zhang et al. 2014, 2016). As the spectrum usually offers a much more detailed picture, it allowed them to explain an earlier report on the net negative helicity of the extremely complex AR 11515, which emerged in the southern hemisphere (Lim et al. 2016). In order to also determine simultaneously the global spectrum, Brandenburg et al. (2017, hereafter BPS17) developed a two-scale formalism that allows us to describe a fairly complex sign rule of solar magnetic helicity, which depends on the position, showing a systematic latitudinal dependence, as well as scale. They applied it to HMI data from three consecutive Carrington rotations (CRs), 2161-2163, and found no evidence of bihelical magnetic fields.

In the present work, we exploit the two-scale approach to determine the solar magnetic helicity spectrum using SOLIS/ Vector Spectromagnetograph (VSM) data from 74 CRs covering more than 6 yr of SC 24. In Section 2 we review some basic definitions and outline the two-scale approach. In Section 3 we discuss the data and error estimation, and in Section 4 we present the magnetic helicity spectra computed at various phases of SC 24. We discuss the implications of our results and conclude in Section 5.

\section{Basic Definitions and Two-scale Approach}

We first recall some fundamental aspects of the relevant physical quantities and then briefly outline the two-scale approach recently developed by BPS17 to determine a global spectrum of magnetic helicity. Let $B_{i}(\boldsymbol{x}, t)$ denote the $i$ th component of the magnetic field, with $t$ being time, $\boldsymbol{x}$ the position vector on the 2D Cartesian surface, and $i=(x, y, z)$. The two-point correlation tensor of the total magnetic field $\boldsymbol{B}(\boldsymbol{x})$ is usually defined as $M_{i j}(\boldsymbol{\xi})=\left\langle B_{i}(\boldsymbol{x}) B_{j}(\boldsymbol{x}+\boldsymbol{\xi})\right\rangle$, which is assumed to be statistically independent of $\boldsymbol{x}$ under homogeneous conditions, where the brackets denote an ensemble average (Batchelor 1953; Moffatt 1978). We omit specifying explicitly the temporal dependencies from now on. The spectrum of magnetic energy, $E_{\mathrm{M}}(k)$, is then given by

$$
2 E_{\mathrm{M}}(k)=\int \delta_{i j} \hat{M}_{i j}(\boldsymbol{k}) k d \Omega,
$$

where

$$
\hat{M}_{i j}(\boldsymbol{k})=\int M_{i j}(\boldsymbol{x}) e^{-i \boldsymbol{k} \cdot \boldsymbol{x}} d^{2} x /(2 \pi)^{2}
$$

is the 2D Fourier transform of $M_{i j}$, the wavevector $\boldsymbol{k}$ denotes the conjugate variable to $\boldsymbol{x}$, and $E_{\mathrm{M}}(k)$ is measured in $\mathrm{G}^{2} \mathrm{~cm}$ rather than $\mathrm{erg} \mathrm{cm}^{-2}$. In two dimensions, $\int d \Omega=2 \pi$ is the circumference of a unit circle, and the integral in Equation (1) is performed over shells in wavenumber space. The scaled magnetic helicity spectrum, $k H_{\mathrm{M}}(k)$, which has the same dimensions as that of $E_{\mathrm{M}}(k)$, is similarly defined as

$$
k H_{\mathrm{M}}(k)=\int i \hat{k}_{i} \epsilon_{i j k} \hat{M}_{j k}(\boldsymbol{k}) k d \Omega,
$$

where $\hat{k}_{i}=k_{i} /|\boldsymbol{k}|$ is the unit vector of $\boldsymbol{k}$ and $k=|\boldsymbol{k}|$ is its modulus with $k^{2}=k_{x}^{2}+k_{y}^{2}$. Thus, the spectra of magnetic energy and helicity can be determined from the two-point 
correlation function using Equations (1) and (3), where the former is given by the trace of the Fourier transform of $M_{i j}$ resulting in a positive-definite scalar quantity, $E_{\mathrm{M}}$, whereas the latter is defined by the skew-symmetric part of $\hat{M}_{j k}$ giving a pseudo-scalar quantity, $H_{\mathrm{M}}$, which can take both positive and negative values (Batchelor 1953; Moffatt 1978; Brandenburg \& Subramanian 2005).

As discussed in BPS17, relaxing the assumption of homogeneity allows us to determine the spectra as a function of a slowly varying coordinate denoted by, say, $\boldsymbol{X}$. This is particularly relevant for the Sun, where we expect opposite signs of helicities in the northern and southern hemispheres, while assuming statistically similar conditions at all longitudes. Below we describe such a procedure to determine spectra that involve a double-Fourier transform.

\subsection{The Two-scale Approach}

Under nonhomogeneous conditions, the two-point correlation function, $M_{i j}\left(\boldsymbol{x}^{\prime}, \boldsymbol{x}^{\prime \prime}\right)=\left\langle B_{i}\left(\boldsymbol{x}^{\prime}\right) B_{j}\left(\boldsymbol{x}^{\prime \prime}\right)\right\rangle$, takes the form (Roberts \& Soward 1975)

$$
M_{i j}(\boldsymbol{X}, \boldsymbol{x})=\left\langle B_{i}\left(\boldsymbol{X}+\frac{1}{2} \boldsymbol{x}\right) B_{j}\left(\boldsymbol{X}-\frac{1}{2} \boldsymbol{x}\right)\right\rangle,
$$

where $\boldsymbol{X}=\left(\boldsymbol{x}^{\prime}+\boldsymbol{x}^{\prime \prime}\right) / 2$ is the mean or slowly varying coordinate and $\boldsymbol{x}=\boldsymbol{x}^{\prime}-\boldsymbol{x}^{\prime \prime}$, called the relative coordinate, is the distance between the two points around $\boldsymbol{X}$. Fouriertransforming Equation (4) first over $\boldsymbol{x}$ and then over $\boldsymbol{X}$ after assuming locally isotropic conditions, one obtains the following simple expression for the doubly Fourier-transformed two-point correlation function (BPS17):

$$
\tilde{M}_{i j}(\boldsymbol{K}, \boldsymbol{k})=\left\langle\hat{\boldsymbol{B}}_{i}\left(\boldsymbol{k}+\frac{1}{2} \boldsymbol{K}\right) \hat{\boldsymbol{B}}_{j}^{*}\left(\boldsymbol{k}-\frac{1}{2} \boldsymbol{K}\right)\right\rangle .
$$

Here the wavevectors $\boldsymbol{K}$ and $\boldsymbol{k}$ denote the conjugate variables to $\boldsymbol{X}$ and $\boldsymbol{x}$, respectively. Analogously to Equations (1) and (3), the $\boldsymbol{K}$-dependent magnetic energy and helicity spectra are thus determined from (BPS17)

$$
\begin{gathered}
2 \tilde{E}_{\mathrm{M}}(\boldsymbol{K}, k)=\int \delta_{i j} \tilde{M}_{i j}(\boldsymbol{K}, \boldsymbol{k}) k d \Omega, \\
k \tilde{H}_{\mathrm{M}}(\boldsymbol{K}, k)=\int i \hat{k}_{i} \epsilon_{i j k} \tilde{M}_{j k}(\boldsymbol{K}, \boldsymbol{k}) k d \Omega .
\end{gathered}
$$

The spectrum of magnetic helicity with a slow variation in the $z$ direction is proportional to $\sin K_{Z} Z$ and is given by $\boldsymbol{K}=\left(0,0, K_{Z}\right)$, where $K_{Z}=2 \pi / L$ and $z=Z$ are used interchangeably.

Unlike $H_{\mathrm{M}}(\boldsymbol{X}, k)$, which is real, $\tilde{H}_{\mathrm{M}}(\boldsymbol{K}, k)$ is complex. The quantity of interest depends on the spatial profile of the background helicity. Rotating and gravitationally stratified bodies such as the Sun are expected to harbor helical flows with a sinusoidal modulation of kinetic helicity as a function of latitude and a change of sign at the equator. This is because the kinetic helicity associated with a perturbed fluid parcel is related to $\boldsymbol{g} \cdot \boldsymbol{\Omega}_{\odot}$, that is, the dot product of gravity $\boldsymbol{g}$ and angular velocity $\Omega_{\odot}$. Note that this dot product changes sign at the equator. Both simulations (see Figure 1(b) of Käpylä et al. 2012) and observations (see Figure 9(b) of Brandenburg et al. 2017) show that the actual helicity profile is more concentrated toward the equator than what is suggested by a simple sinusoidal profile and that contributions from $K>K_{0}$ could therefore become relevant. However, most crucial for the purpose of this paper is the sign change at the equator. Furthermore, we do not expect any systematic variation or a sign change of helicity in the longitudinal direction. This is because the Sun is predominantly axisymmetric concerning both its velocity and magnetic structures. If turbulence was driven by the magnetic buoyancy instability, spontaneous helicity production of either sign is in principle possible (Chatterjee et al. 2011a). This can perhaps account for occasional departures from a strict north-south antisymmetry, which we still expect to prevail on average. Indeed, numerical simulations of BPS17 demonstrated that the magnetic helicity of a helically driven turbulent dynamo reflects a similar sinusoidal variation along $Z$ when the background turbulence possesses kinetic helicity that varies sinusoidally with $Z$, changing sign at the equator at $Z=0$.

Following the motivation laid out above, we focus here on helicity profiles proportional to $\sin K_{0} Z$ with the equator at $Z=0$. Its Fourier transform is $-\frac{1}{2} i \delta\left(K_{Z}-K_{0}\right)$. We will therefore plot the negative imaginary part of $\tilde{H}_{\mathrm{M}}(\boldsymbol{K}, k)$, which reflects the sign of magnetic helicity in the northern hemisphere. The total magnetic energy $\mathcal{E}_{\mathrm{M}}$ and helicity $\mathcal{H}_{\mathrm{M}}\left(K_{0}\right)$ are defined as

$$
\begin{gathered}
\mathcal{E}_{\mathrm{M}}=\int_{0}^{\infty} \tilde{E}_{\mathrm{M}}(0, k) d k, \\
\mathcal{H}_{\mathrm{M}}\left(K_{0}\right)=\int_{0}^{\infty} \tilde{H}_{\mathrm{M}}\left(K_{0}, k\right) d k,
\end{gathered}
$$

which will be used in Section 4.

\section{Data Analysis}

We analyze synoptic vector magnetograms from 74 CRs of the Sun where we determine the magnetic energy and helicity spectra either for each CR or by sometimes first combining the synoptic vector magnetograms from three successive CRs. The data are based on measurements from the VSM instrument on the SOLIS project (Keller et al. 2003; Balasubramaniam \& Pevtsov 2011). SOLIS/VSM observes Fe I 630.15 and $630.25 \mathrm{~nm}$ spectral lines, with a spatial sampling of 1 !" 14 per pixel and a $2048 \times 2048$ pixel field of view. The line profiles of Stokes $Q, U, V$, and $I$ are derived using the Very Fast Inversion of the Stokes Vector code (Borrero et al. 2011) on the Fe I $630.25 \mathrm{~nm}$ line, which includes the magnetic field filling factor. The $180^{\circ}$ ambiguity in the transverse field direction is solved using the Very Fast Disambiguation Method (Rudenko \& Anfinogentov 2014). Synoptic maps of the three vector components of the photospheric magnetic field are constructed from daily full-disk magnetograms. We use the $180 \times 360$ pixel maps of the photospheric vector magnetic field, where each pixel gives the observed full vector magnetic field $\boldsymbol{B} \equiv\left(B_{r}, B_{\theta}, B_{\phi}\right)$, with $r, \theta$, and $\phi$ corresponding to the radius, colatitude, and longitude, respectively. The field is mapped onto the $(\phi, \mu)$ plane with $\mu=\cos \theta$, allowing us to adopt a righthanded Cartesian analysis by substituting

$$
(\phi, \mu) \rightarrow(y, z), \quad\left(B_{r}, B_{\phi},-B_{\theta}\right) \rightarrow\left(B_{x}, B_{y}, B_{z}\right) .
$$

In the definitions of quantities of interest, notably $k \tilde{H}_{\mathrm{M}}(\boldsymbol{K}, k)$, given in Section 2.1, we consider a fixed wavevector $\boldsymbol{K}=\left(0, K_{0}\right)$, where $K_{0}=\pi / R_{\odot}$, with $R_{\odot}$ being the solar radius, and determine this as a function of $k$. Thus, as noted before, we assume that the background helicity does not have any systematic modulation in longitude. For the energy 
spectrum, we consider no modulation, as usual, and determine $\tilde{E}_{\mathrm{M}}(0, k)$ versus $k$.

We consider all CR numbers between 2093 and 2178, except CRs 2099, 2107, 2127, 2139, 2152-2155, 2163, 2164, 2166, and 2167. These rotations suffer from poor data coverage and therefore depict obvious outliers. Our analysis thus covers a period from 2010 January 30 to 2016 July 3 of the current SC 24, which reached its maximum during the middle of the year 2014. The wavelength-dependent scatter of the spectra can be considered as a measure of the error introduced by the temporal evolution of the synoptic maps and, to a smaller extent, stochastic errors in the measurement of the magnetic field vector. We define the rms error $\sigma_{\mathcal{P}}(k)$ associated with the spectrum $\mathcal{P}(k)$ as

$$
\sigma_{\mathcal{P}}(k)=\sqrt{\left\langle\left(\mathcal{P}(k)-\langle\mathcal{P}(k)\rangle_{\mathrm{CR}}\right)^{2}\right\rangle},
$$

where \langle\rangle$_{\mathrm{CR}}$ denotes the average over CRs 2148-2151, which corresponds to the period of maximum solar activity. The statistical error adopted here is expected to be largest at this phase, and therefore it is safer to read the spectra even from other epochs in the light of overestimated errors being shown.

This error, however, does not contain the uncertainty of the magnetic field measurement itself. Noise in spectral line observations, uncertainties and simplifications in inversion method (like assumption of a Milne-Eddington-type atmosphere), and possible errors in disambiguation method introduce uncertainties. It is virtually impossible to reliably quantify this error (see, e.g., Borrero et al. 2014). Moreover, the synoptic map is constructed from consecutive observations over solar rotation; it is not a snapshot. Averaging over a large number of pixels observed within a few days improves the signal-to-noise ratio but makes the variable small-scale magnetic features less reliable.

\section{Results}

\subsection{Average Spectrum during the Maximum of SC 24}

First we show, in Figure 2, the spectra of magnetic helicity and energy as a function of $k$, obtained after averaging over individual spectra from CRs 2148-2151, which correspond to the phase when the Sun was most active during SC 24 in terms of sunspot number. As noted before, the relevant quantity here is $-\operatorname{Im} k \tilde{H}_{\mathrm{M}}\left(K_{0}, k\right)$, which has the sign convention corresponding to the northern hemisphere. Remarkably, the averaged spectrum of solar magnetic helicity, denoted by $\left\langle-\operatorname{Im} k \tilde{H}_{\mathrm{M}}\left(K_{0}, k\right)_{\mathrm{CR}}\right\rangle$, as shown in the top panel of Figure 2, clearly reveals a bihelical signature, with positive (negative) helicity at small (large) wavenumbers, exactly as would be expected from an $\alpha$ effect-driven solar dynamo (Blackman \& Brandenburg 2003; Yousef \& Brandenburg 2003); see Figure 1 for the expected HSR. However, the power at small $k$ is rather weak. The $k$-dependent errors, $\sigma_{2 E}$ and $\sigma_{k H}$, estimated according to Equation (11), are shown in the bottom panel of Figure 2. The retrieved magnetic helicity spectrum during the maximum phase of SC 24 is significant at all $k$ when contrasted with its error.

In the context of the solar dynamo, a distinction between large and small scales can be made based on the wavenumber $k$, where the magnetic helicity changes sign, i.e., at $k \approx$ $0.02 \mathrm{Mm}^{-1}$, corresponding to a scale $\mathcal{L} \approx 315 \mathrm{Mm}$ from Figure 2. Typical scales associated with ARs of $\sim 30 \mathrm{Mm}$ are therefore considered "small," whereas the large scales are
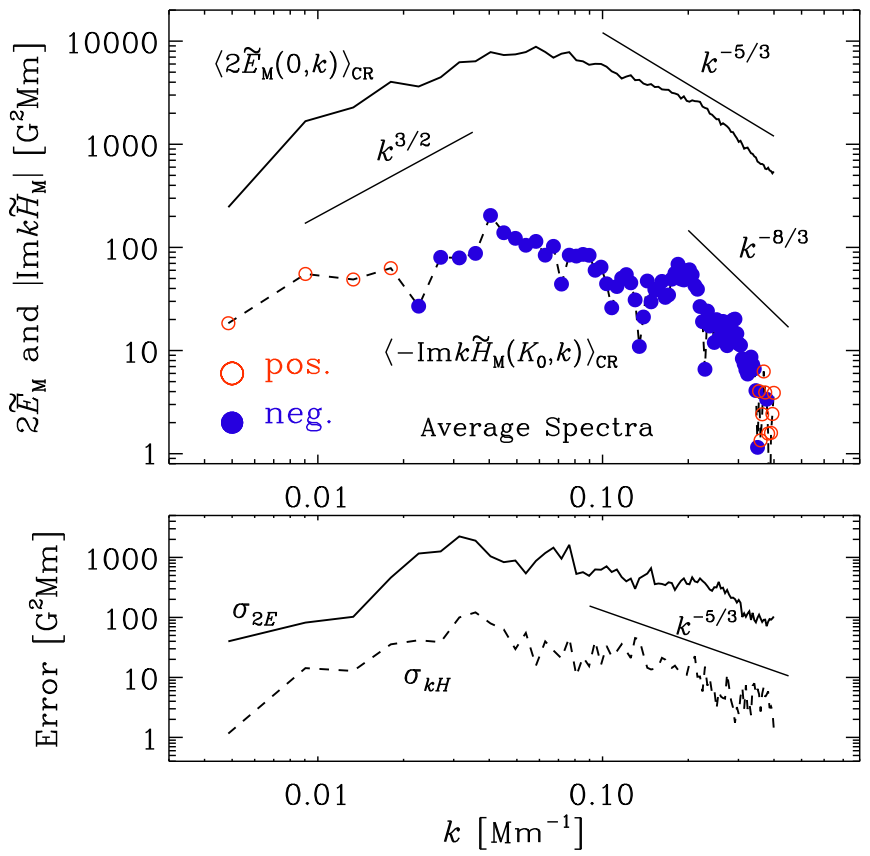

Figure 2. Top: magnetic energy (solid line) and helicity (dashed line, circles) spectra obtained after averaging spectra from CRs 2148-2151. Sign convention adopted here corresponds to the sign of magnetic helicity in the northern hemisphere; open red (filled blue) circles denote positive (negative) signs for the magnetic helicity. Bottom: errors on $\left\langle 2 \tilde{E}_{\mathrm{M}}\right\rangle_{\mathrm{CR}}$ and $\left\langle-\operatorname{Im} k \tilde{H}_{\mathrm{M}}\right\rangle_{\mathrm{CR}}$, as determined from Equation (11), are shown by solid and dashed lines, respectively.

comparable to the solar radius. We also note that such a distinction is not always possible, as the spectrum shows significant variation between different epochs. Nevertheless, it gives us a perspective on the relevant scales involved in the underlying dynamo mechanism.

We recall that an energy spectrum proportional to $k^{2}$ (Saffman spectrum) means that the large-scale field is random. However, only the steeper $k^{4}$ Batchelor spectrum would imply that the largest scales are not causally related to the smaller ones (Durrer \& Caprini 2003). All the spectra retrieved in this study show shallower power laws at the smallest wavenumbers, implying a causal connection between the large and small scales. As discussed further below, it is also possible that we see evidence of Kazantsev scaling with a $k^{3 / 2}$ subrange at $k \mathrm{Mm}<0.03$, which would be indicative of a small-scale dynamo (SSD; Kazantsev 1968). Indeed, astrophysical dynamos operating at high magnetic Reynolds numbers are expected to exhibit a unified version of dynamo action that combines elements of both SSDs and LSDs (Subramanian 1999; Subramanian \& Brandenburg 2014; Bhat et al. 2016). On the other hand, the bihelical magnetic field expected from an $\alpha$ effect is usually expected to imply an actual increase in magnetic power at small $k$; see Figure 3 of Brandenburg (2001). All the spectra computed here show less power at small wavenumbers than in the large ones; this could be a manifestation of the SSD dominating the LSD near the surface (Trujillo Bueno et al. 2004).

At the largest values of $k$, one also sees occasional data points with a reversed sign, but the power is again low. The measured power is dropping below its estimated error at wavenumbers where the sign change occurs, so this cannot be regarded as a reliable finding. Nevertheless, the occurrence of mixed signs as such is not surprising given the turbulent nature 


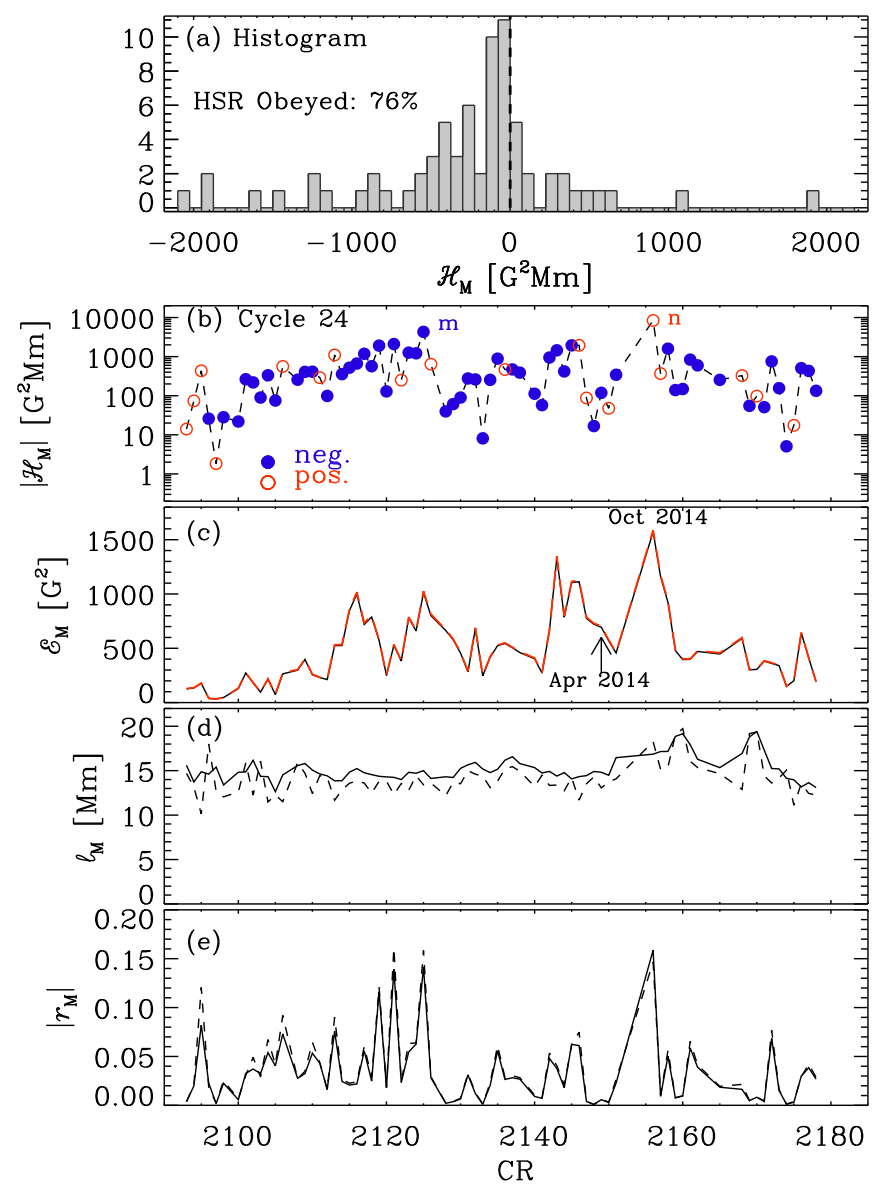

Figure 3. (a) Histogram of $\mathcal{H}_{\mathrm{M}}$, and temporal evolution of (b) $\left|\mathcal{H}_{\mathrm{M}}\right|$, (c) $\mathcal{E}_{\mathrm{M}}$, (d) $\ell_{M}$, and (e) $\left|r_{M}\right|$; see Equations (8), (9), (12), and (13). The blue filled (red open) circles in panel (b) denote, as usual, negative (positive) values, with sign convention corresponding to the northern hemisphere. The two largest values of $\left|\mathcal{H}_{\mathrm{M}}\right|$ marked by letters "m" and "n" are ignored in the histogram. Solid black and dashed red curves in panel (c) correspond to $\mathcal{E}_{\mathrm{M}}$ from Equation (8) and directly from synoptic maps, respectively. Dashed and solid lines in panels (d) and (e) are based on original and smoothed spectra using a three-point boxcar filter, respectively.

of the underlying magnetic field and has been seen before (BPS17). However, compared with the usual one-scale approach used in Zhang et al. (2014, 2016), these mixed signs are surprisingly rare.

\subsection{HSR Statistics}

Before discussing more spectra from a few individual CRs at different phases of SC 24, we next look at the temporal evolution of the total integrated magnetic energy $\mathcal{E}_{\mathrm{M}}$ and helicity $\mathcal{H}_{\mathrm{M}}$, defined in Equations (8) and (9), respectively. These are determined by first obtaining the corresponding spectra for each CR using Equations (6) and (7) and then computing the $k$ integral. The integrated magnetic helicity is shown in Figures 3(a) and (b), which reveals that it is generally small, as might be expected for bihelical magnetic fields leading to significant cancellations of opposite helicities at large and small scales. As is evident from the histogram presented in Figure 3(a), the most common values are around a few tens of $\mathrm{G}^{2} \mathrm{Mm}$, while the distribution also develops wide wings with values of the order of 1000 or $2000 \mathrm{G}^{2} \mathrm{Mm}$, but such events are relatively rare. They can be associated with complex ARs dominating the spectrum with significant intrinsic magnetic helicity. We discuss some examples later in this paper.

The median of the distribution is clearly negative, as can also be seen in the dominance of blue circles in Figure 3(b). This is due to the large-scale contributions, giving a positive signal in the northern hemisphere if HSR is obeyed, being subdominant to the negative helicity carried by the ARs. Therefore, positive values of this quantity can indicate either an occasionally dominating positive large-scale contribution or a non-HSRobeying positive helicity at the smaller scales. The former happens only during the early declining phase of SC 24 , when magnetic energy and helicity obtain maxima, as will be discussed in detail in Section 4.4. Therefore, a negative (positive) sign of the integrated magnetic helicity can be regarded as a good proxy obedience (violation) of HSR.

Of all the $74 \mathrm{CRs}$ analyzed, $76 \%$ exhibit negative integrated magnetic helicity and are therefore judged to obey the HSR. Note again that we follow here the sign convention of the northern hemisphere, which may be inferred from Figure 1. The likelihood to obey HSR is increased during the ascending phase of the SC, while it is decreased during the first few CRs of SC 24. The integrated magnetic energy, shown in Figure 3(c), attains a maximum in 2014 October, when also very large magnitudes of magnetic helicity are seen. This is roughly 6 months later than the maximum of SC 24 obtained from sunspot numbers (2014 April). Already before the maximum energy and helicity are reached, the sign of the integrated magnetic helicity becomes ill-defined, the reasons behind this being discussed in Section 4.3, and this behavior continues during the declining phase; see Section 4.4.

It is useful to have some estimate of the integral scale of turbulence $\ell_{M}$, which is defined as

$$
\ell_{\mathrm{M}}=\int_{0}^{\infty} k^{-1} \tilde{E}_{\mathrm{M}}(0, k) d k / \int_{0}^{\infty} \tilde{E}_{\mathrm{M}}(0, k) d k .
$$

We show in Figure 3(d) the time evolution of $\ell_{M}$ based on the original as well as smoothed spectra using a three-point boxcar filter. Its average, $\ell_{\mathrm{M}} \approx 15 \mathrm{Mm}$, is somewhat smaller than a previous estimate by BPS17 $\left(\ell_{\mathrm{M}} \approx 20 \mathrm{Mm}\right)$. This discrepancy is likely a result of the higher spatial resolution of the HMI data used by BPS17. We know from the realizability condition (Moffatt 1978; Kahniashvili et al. 2013), i.e., $\left|\mathcal{H}_{\mathrm{M}}\right| / 2 \mathcal{E}_{\mathrm{M}} \leqslant \ell_{\mathrm{M}}$, that the magnetic energy of helical fields is bounded from below, and therefore the absolute value of the quantity

$$
r_{\mathrm{M}}=\mathcal{H}_{\mathrm{M}} / 2 \ell_{\mathrm{M}} \mathcal{E}_{\mathrm{M}}
$$

cannot exceed unity. In Figure 3(e), we show the evolution of $\left|r_{\mathrm{M}}\right|$ and note that the realizability condition is obeyed at all times, with $\left|r_{\mathrm{M}}\right|$ being always below 0.2 . This is similar to what was obtained in BPS17. It should be noted that the integral scale in Equation (12) is sometimes defined with a $2 \pi$ factor. We have ignored this here to avoid a corresponding factor in Equation (13). However, the actual energy-carrying structures tend to be larger by a similar factor.

It is also important to check how well the HSR proxy, based on the total magnetic helicity, works by inspecting how the sign of magnetic helicity changes at a few selected values of $k$. With the sign convention of the northern hemisphere as before, we show in Figure 4 the temporal evolution of $2 \tilde{E}_{\mathrm{M}}$ and $-\operatorname{Im} k \tilde{H}_{\mathrm{M}}$ at three fixed values of $k$. Again, most of the analyzed data reveal that the expected HSR is obeyed, as may 

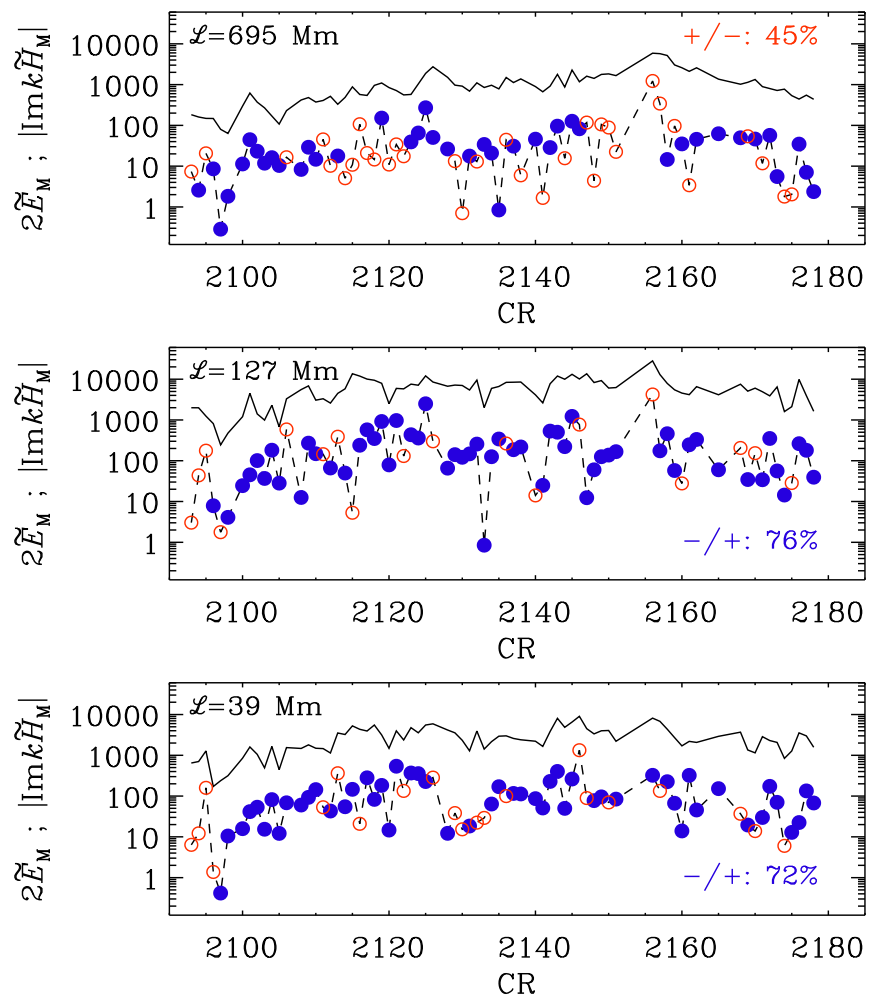

Figure 4. Temporal evolution of spectral magnetic energy and helicity at $k \approx 0.01 \mathrm{Mm}^{-1}$ (top), $0.05 \mathrm{Mm}^{-1}$ (middle), and $0.16 \mathrm{Mm}^{-1}$ (bottom), where corresponding length scale $\mathcal{L}=2 \pi / k$ is displayed in panels; " $+/-$ " (" $-/+$ ") denotes the percentage fraction of positive (negative) sign of the magnetic helicity at chosen $k$.

be seen from the bottom two panels corresponding to intermediate and small scales, dominated mostly by the ARs, which are expected to carry a net negative helicity in the north. However, the top panel corresponding to large scales shows a larger fraction of CRs violating the HSR. The absolute values of the helicity are indeed much smaller at these scales, and better estimates are therefore needed to reliably determine the sign of magnetic helicity at large scales.

\subsection{Early Rising Phase of SC 24}

We now show in Figure 5 the spectra of magnetic helicity and energy that are obtained after stitching together data from three consecutive CRs 2093-2095, which correspond to the early rising phase of SC 24 covering a period from 2010 January 30 to 2010 April 21. The corresponding synoptic maps of only the radial component of the magnetic field, $B_{r}$, are also shown in Figure 5. Note that with $\phi$ being longitude, the range $0^{\circ} \leqslant \phi<360^{\circ}$ refers to $\mathrm{CR} 2095,360^{\circ} \leqslant \phi<720^{\circ}$ refers to CR 2094, and $720^{\circ} \leqslant \phi \leqslant 1080^{\circ}$ refers to CR 2093.

The magnetic energy and helicity peak at smaller scales, approximately at 0.07 and $0.09 \mathrm{Mm}^{-1}$, than during the maximum phase, but they obtain similar magnitudes to those during the maximum at their peak values. The large-scale powers are very weak and fall below the estimated errors. The spectral scaling is steep, close to the Saffman spectrum with $k^{2}$, indicating random large-scale fields, but due to the weak signal, large uncertainty is related to this value.

Although the magnetic fields are clearly bihelical, the signs of magnetic helicity at small and large $k$ are exactly opposite of what we expect from a simplistic turbulent dynamo model.
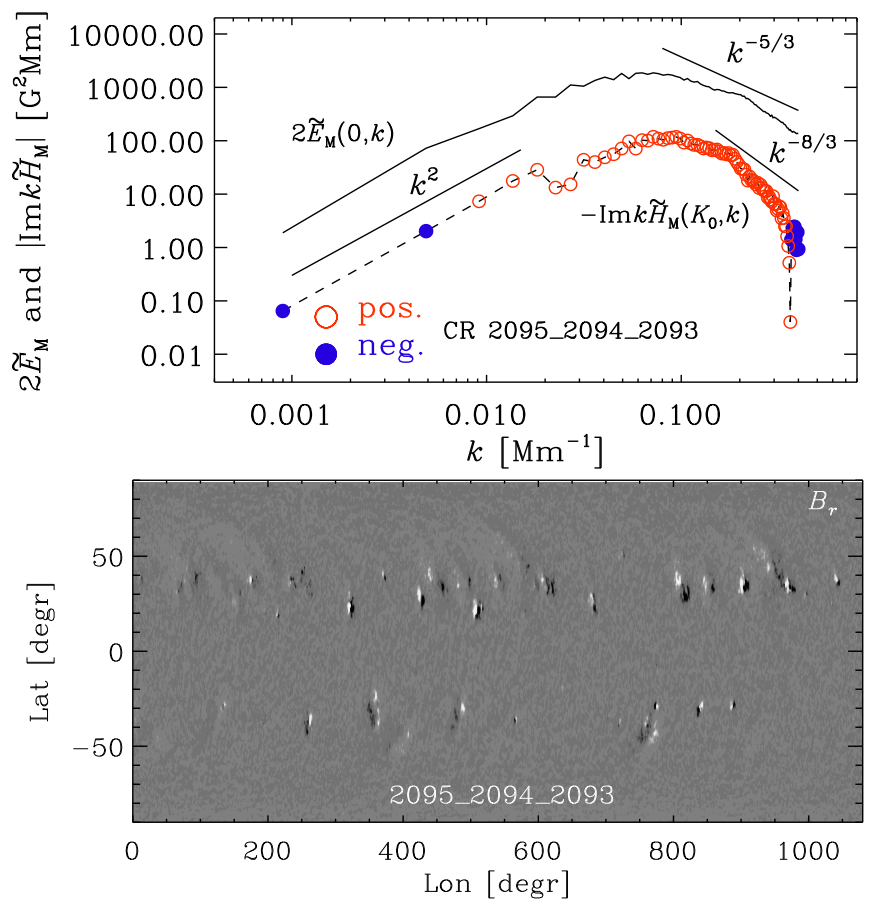

Figure 5. Top: magnetic helicity and energy spectra from the interval spanning CRs 2093-2095. Spectra are determined after stitching together data from these CRs. Bottom: synoptic chart of the radial component of the magnetic field, $B_{r}$, covering the same time span. The sign convention adopted here corresponds to the sign of magnetic helicity in the northern hemisphere; open (filled) circles denote positive (negative) signs for the magnetic helicity.

Choudhuri et al. (2004), however, discussed a different (Babcock-Leighton-type) dynamo model that can predict such violations of HSR during the early phase of the cycle. These arise due to the flux tubes of the new cycle emerging in regions where poloidal fields from the previous cycle, possessing helicity of a wrong sign, still persist. Turbulent dynamo models can also produce similar sign reversals when magnetic helicity conservation law is used to constrain the model (Pipin et al. 2013).

In comparison to other observational results, current helicitybased proxies indicate such reversals for SC 22 (Bao et al. 2000; Zhang et al. 2010), while not for SC 24 (Gosain et al. 2013), and the results for SC 23 remain contradictory, Pevtsov et al. (2001) against and Zhang et al. (2010) in favor of reversals. In contrast, the Pipin \& Pevtsov (2014) results computing the global magnetic helicity using azimuthally averaged mean magnetic field indicate a sign reversal at large scales in the early phases of SC 24.

From the magnetogram showing the radial magnetic field $B_{\mathrm{r}}$ in Figure 5, we see that most ARs are located at higher latitudes, as expected if the ARs followed the butterfly diagram typical for the early rising phase, and therefore we do not expect significant "leakage" of magnetic helicity of opposite sign through the equator. It appears more likely that the ARs are intrinsically twisted in an opposite sense and dominate the magnetic helicity spectrum in Figure 5 with small-scale positive helicity in the northern hemisphere, showing a maximum at scales around $60 \mathrm{Mm}$.

\subsection{Early Declining Phase of SC 24}

Similarly, in Figure 6 we show the spectra and the magnetogram from the interval spanning CRs 2156-2158, 

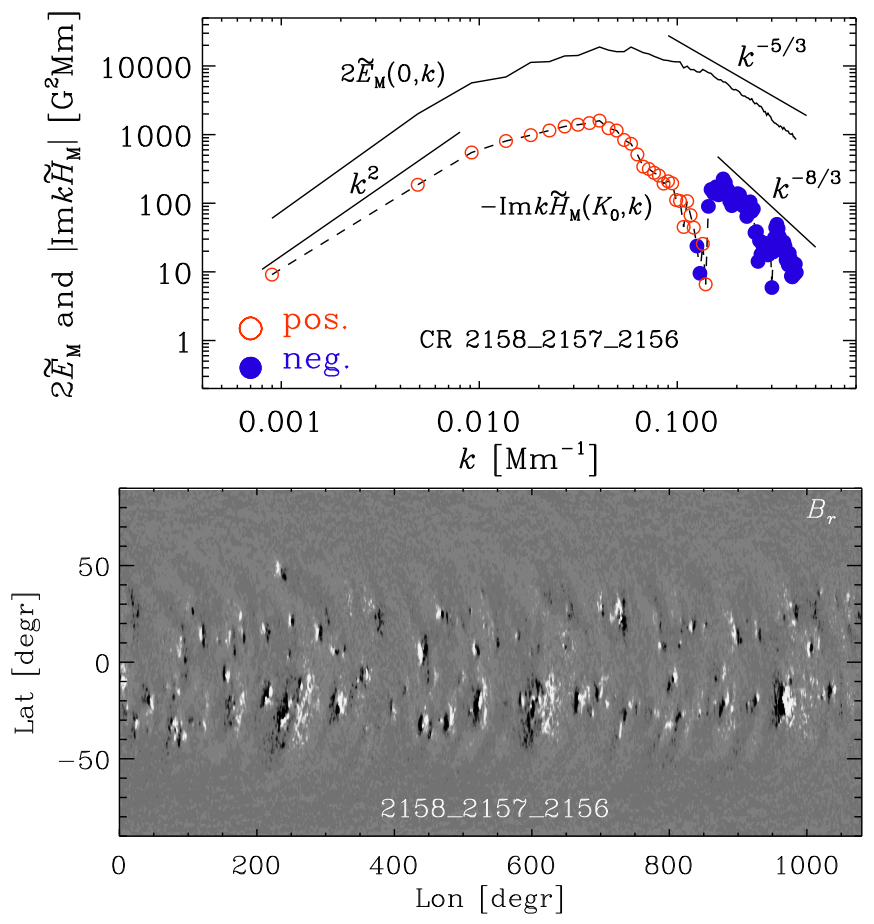

Figure 6. Same as Figure 5, but for the interval spanning CRs 2156-2158.

which corresponds to the period from 2014 October 15 to 2015 January 3, i.e., just after the maximum of SC 24 . While SC 24 reached its maximum in terms of the actual number of sunspots in 2014 April, the energy and also the helicity show a peak during 2014 October corresponding to CR 2156; see Figure 3. The magnetic helicity spectrum now once again shows a bihelical signature with signs at small and large $k$ being compatible with the HSR based on an $\alpha$-driven solar dynamo. This is qualitatively similar to the averaged helicity spectrum shown in Figure 2, but we also note an important difference. Here we find a positive sign for the peak value of $-\operatorname{Im} k \tilde{H}_{\mathrm{M}}\left(K_{0}, k\right)$ at $k_{\text {peak }} \approx 0.04 \mathrm{Mm}^{-1}$, i.e., scales around $\mathcal{L}_{\text {peak }} \approx 160 \mathrm{Mm}$, with the spectrum turning negative for $k>0.1 \mathrm{Mm}^{-1}$, i.e., at scales smaller than $60 \mathrm{Mm}$. Hence, while the magnetic helicity spectrum at the largest and very smallest scales remains largely unaltered, in the midrange scales, where usually the ARs dominate with strongly negative helicity, we observe strong reversed (i.e., positive) magnetic helicities. As a result, the total solar magnetic helicity $\mathcal{H}_{\mathrm{M}}$ during this period is positive in the northern hemisphere (marked by the letter "n" in the inset of Figure 3(b)), thus appearing to violate the HSR, defined based on the sign of the total magnetic helicity.

However, a closer look presents a much richer picture accessible only through a spectrum such as the ones being explored here. Comparing the integral scale of turbulence $\ell_{\mathrm{M}} \approx 20 \mathrm{Mm}$ as noted below Equation (12) to $\mathcal{L}_{\text {peak }}$ determined above gives a scale separation $\zeta=\mathcal{L}_{\text {peak }} / \ell_{\mathrm{M}} \approx 8$. Assuming this to be sufficient for distinguishing between the large and small scales, we let, in this case, $\mathcal{L}_{\text {peak }}$ represent the "large" scale. Then, the helicity spectrum in Figure 6 is reminiscent of a classic picture due to an LSD where the spectra have a peak at scales that are considerably larger than the turbulent scales. Interestingly enough, Sheeley \& Wang (2015) found that the Sun's large-scale magnetic field was rejuvenated exactly during this period. This is further supported by our inference that

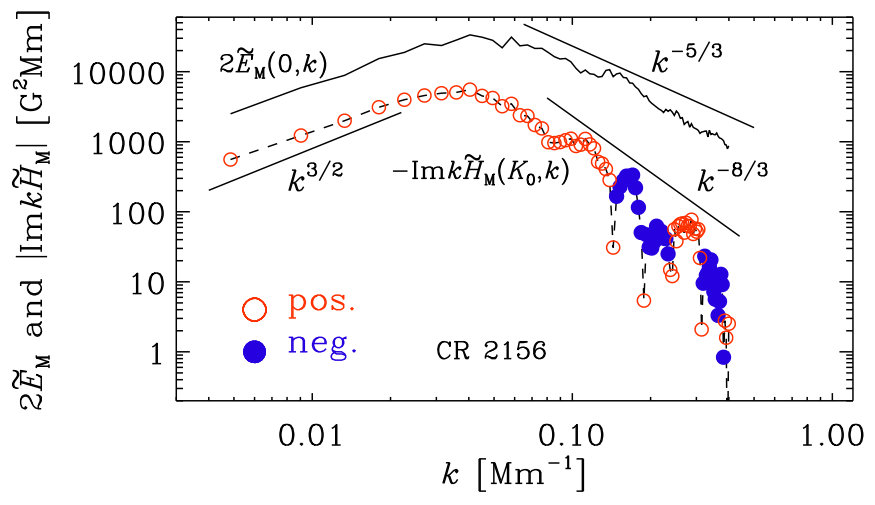

Figure 7. Same as Figure 2, but for CR 2156, which corresponds to the time when both magnetic energy and helicity reach a maximum in Figure 3.

the power spectra are dominated by the LSD during CRs 2156-2158, thus resulting in positive $\mathcal{H}_{\mathrm{M}}$ in the northern hemisphere without, it seems, violating the HSR.

To examine in more detail the epoch when both total magnetic energy and helicity maximize, we zoom into CR 2156 and compute the spectra for it alone (hence the shorter $k$-range) in Figure 7, which, except for showing sign fluctuations in $-\operatorname{Im} k \tilde{H}_{\mathrm{M}}$ at large $k$, looks otherwise similar to Figure 6. The small-scale sign fluctuations might also be caused by many complex ARs, such as 12192, 12205, 12209, 12241,12242 , etc., being, at times, of the $\delta$-type, that could carry intrinsic helicities that are not necessarily always according to the sign rule. For SC 23 , the number of complex ARs was found to decline slower than the total number of ARs, due to which their relative fraction was observed to be higher during the declining phase (Jaeggli \& Norton 2016), which lends support to this scenario. We note, in addition, that the power in the largest scales is significantly enhanced during this $\mathrm{CR}$, an indication of enhanced LSD during this epoch.

Intriguingly, the magnetic energy spectrum shows a Kazantsev scaling of $k^{3 / 2}$, which is predicted for the SSD, albeit for the subinertial range. Here this scaling is, rather unexpectedly, seen at the large scales. As we elaborate in Section 5, these results are suggestive of both LSD and SSD being operative simultaneously in the Sun, with the $k^{3 / 2}$ scaling due to the SSD and bihelicity of fields due to the LSD from an $\alpha$ effect.

\subsection{Late Declining Phase of SC 24}

During the later part of the declining phase, the magnitudes of total magnetic energy and helicity decrease, and the helicity sign shows fluctuations, as can be seen from Figure 3(b). In Figure 8, we show that the spectrum of solar magnetic helicity is very complex during this time epoch. It shows multiple sign reversals as a function of $k$. During CRs 2168-2170, the dominant sign of magnetic helicity in the north is positive, thus violating the HSR. Moreover, sign changes at $k<0.03 \mathrm{Mm}^{-1}$ reflect possible fluctuations at the largest length scales, and it is not necessarily caused by ARs. These sign changes possibly occur owing to spectral power being proportional to $k^{2}$ expected for random fields that are $\delta$-correlated in space.

However, the violation of the sign rule seen at intermediate to large $k$ in the top panel of Figure 8 could indeed be caused by the emergence of some peculiar ARs. To investigate this further, we focus on AR 12443, which emerged close to the equator during CR 2170. This developed a complex $\beta \gamma \delta$-type 

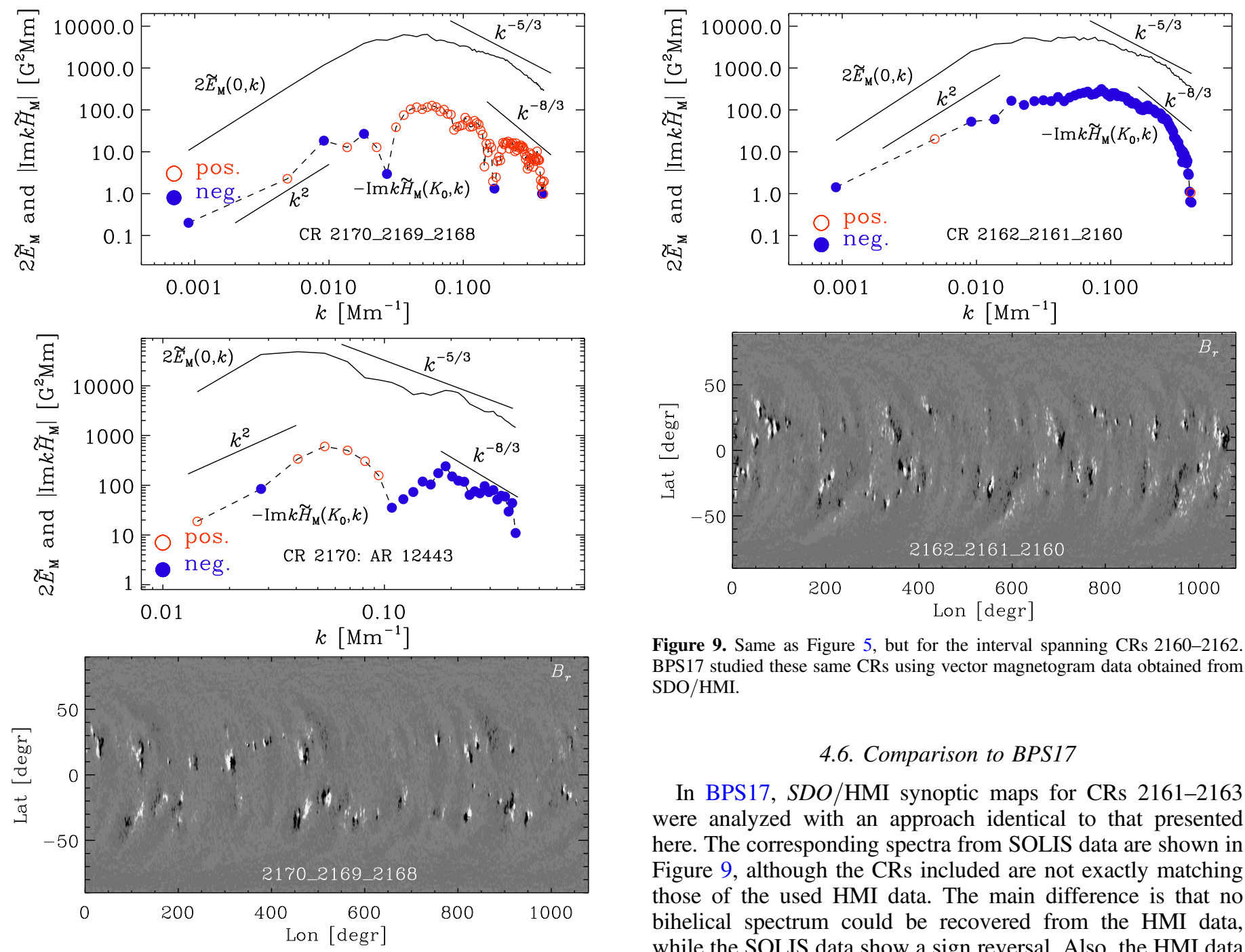

Figure 9. Same as Figure 5, but for the interval spanning CRs 2160-2162. BPS17 studied these same CRs using vector magnetogram data obtained from SDO/HMI.

\subsection{Comparison to BPS17}

In BPS17, SDO/HMI synoptic maps for CRs 2161-2163 were analyzed with an approach identical to that presented here. The corresponding spectra from SOLIS data are shown in Figure 9, although the CRs included are not exactly matching those of the used HMI data. The main difference is that no bihelical spectrum could be recovered from the HMI data, while the SOLIS data show a sign reversal. Also, the HMI data have considerably higher spatial resolution, and therefore the data extend to far larger values of $k$ with better-established power laws, while the SOLIS data fail to show clear power laws. In both computations, we see fluctuations at the largest wavenumbers, possibly due to low-amplitude, small-scale magnetic fields.

To hunt down the reason for the difference seen at small structure and gave rise to a couple of M-class and several C-class flares. The spectra determined from a smaller 2D patch containing AR 12443 are shown in the middle panel of Figure 8, demonstrating that this AR carries, unexpectedly, a net positive magnetic helicity. The proximity of this AR to the equator could be yet another possible reason for the observed violation, as it is not clear whether the underlying LSD activity belt is strictly symmetric about the equator. As discussed in Section 4.2, and as shown by Chatterjee et al. (2011b), this could be a result of spontaneous helicity production of either sign by the magnetic buoyancy instability. Violations of the sign rule, however, are also seen during the very early ascending phase, when sunspots occur at high latitudes. This indicates that the equatorial proximity of the ARs is not a necessary condition for sign rule violations. A systematic study of the relation of AR latitudes and complexities to the magnetic helicity evolution is required to decide on the relevance of these factors; this will be addressed in a future study. Also, more dedicated numerical work is needed in this direction to explore whether such sign anomalies are indeed associated with the morphological complexities of ARs. wavenumbers, we transform back from $K$ space to $Z$ space and show the spectra as a function of latitude in Figure 10. By comparing this figure with Figure 9 of BPS17, we see that there is a good agreement between the results at intermediate scales. The retrieved extrema of the magnetic helicity are larger for SOLIS than for HMI, with the magnetic energy values being in fair agreement. At the small wavenumber end (largest scales), however, differences are obvious. At the smallest $k$, the HMI data show relatively strong signals extending to high latitudes, violating the HSR especially in the high southern latitudes and northern lower latitudes. Similarly, the SOLIS signal at the smallest $k$ comes from higher latitudes. The power at these scales is lower than in the HMI spectra, and the sign rule is not violated as strongly. In both the SOLIS and HMI data, the helicity and magnetic energy fade off at high $k$, and both seem to indicate that north and south are somewhat asymmetric, with the north decreasing more rapidly than the south. In conclusion, the intermediate scales seem to be in fair agreement with both data sets, but some differences can be seen especially at the 

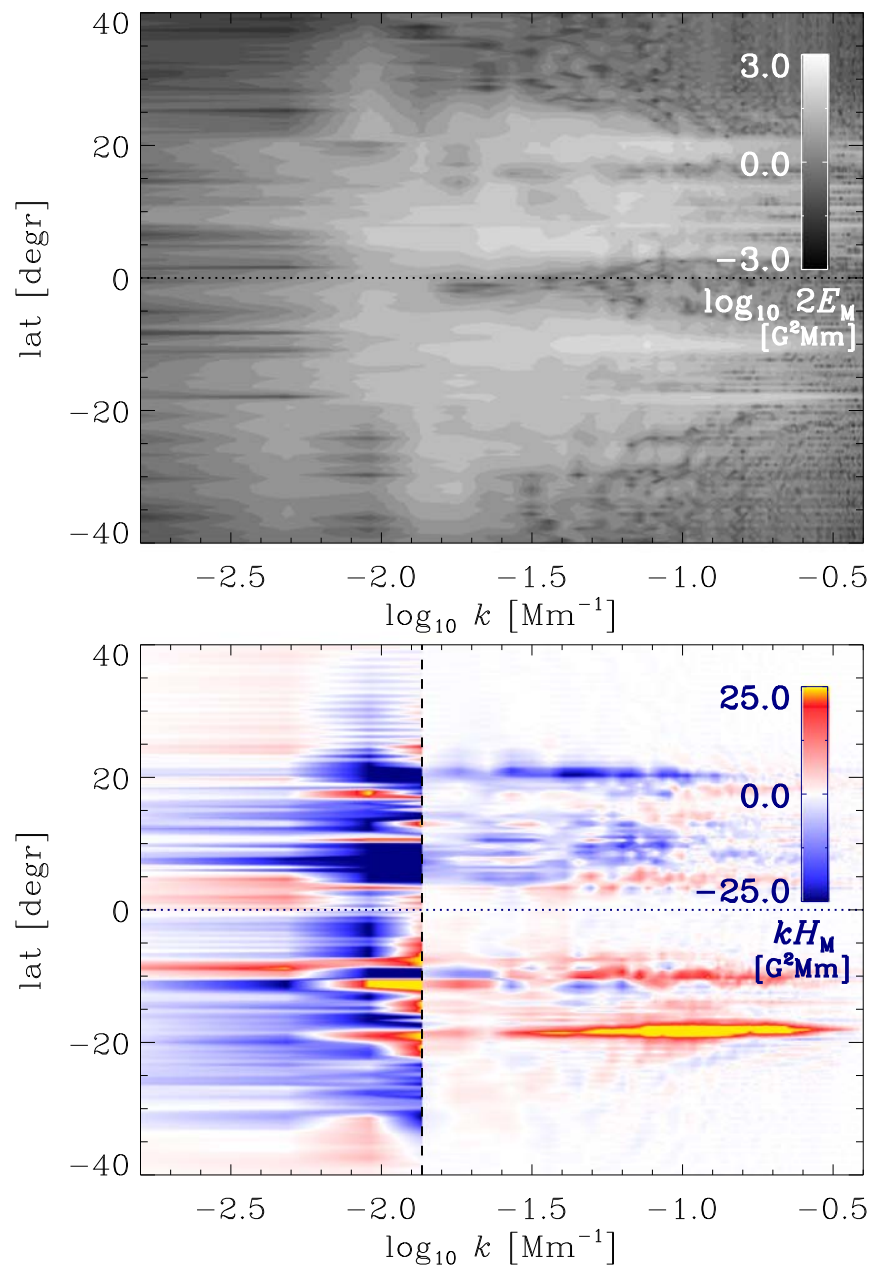

Figure 10. Magnetic energy and helicity spectra for 2D solar surface data for CRs 2160-2162 as a function of $k$ and latitude. Colors to the left of the dashed vertical line are saturated at levels $\pm 2 \mathrm{G}^{2} \mathrm{Mm}$ to highlight the sign reversal in the southern hemisphere.

largest scales. Also, the sign change at low wavenumbers in the southern hemisphere as apparent from Figure 10 was not seen by BPS 17.

Some of the discrepancies might relate to the differences between the two instruments. SOLIS/VSM observations used for this analysis have a significantly higher signal-to-noise ratio and spectral resolution compared to HMI (see, e.g., Thalmann et al. 2012). Recent studies also show that SOLIS and HMI observations of meridional and zonal magnetic fields often disagree at intermediate field strengths (plage regions). This most likely relates to fundamental limitations in Zeeman-effectbased vector magnetic field observations due to the unequal noise in the transverse and line-of-sight components of the magnetic field. HMI data processing also suffers from a lack of a realistic filling factor, which most likely overestimates transverse fields outside ARs (private communication with SOLIS/VSM and SDO/HMI teams).

\section{Discussion}

This study was motivated by the earlier paper by BPS17, where no bihelical magnetic helicity spectra could be retrieved from HMI/SDO data for three CRs during SC 24. Instead, the helicity spectrum showed the same sign at large and small scales, inconsistent with the expectations from a helically driven $\alpha$ effect dynamo scenario. BPS17 argued that this might simply be due to the epoch of the observations having been unfortunate. Another line of thought was that the solar surface could be a special place in between the dynamo-active convection zone and the solar wind. These regions are expected to show reversed signs of helicities according to models (Warnecke et al. 2011, 2012) and solar wind observations (Brandenburg et al. 2011), the sign change possibly occurring in the surface regions, resulting in undetectable or weak systematic helicity signatures. Our current study shows that both lines of thought were partially correct. In fact, more realistic modeling now suggests that the sign change is expected to occur at a height of just $\sim 5 \mathrm{Mm}$ above the surface (Bourdin et al. 2018).

Throughout the nearly $7 \mathrm{yr}$ of data analyzed here, the power at large scales is persistently weaker than that in the midrange scales, distinctively different from the dynamo simulations, where the large scales possess the largest power. This indicates that the helicity signatures of the LSD are, indeed, weak near the surface, overwhelmed by the helicity signal that the ARs carry, influenced by the SSD, and, perhaps most importantly, prone to be affected by noise and any uncertainties related to the data analysis procedures. Our analysis reveals that the expected bihelical signature can be retrieved easily from timeaveraged spectra as computed from the high signal-to-noise SOLIS/VSM synoptic maps, but it also highlights the need for better synoptic maps, covering a significant fraction of the SC, allowing us to find the opposite sign of helicity at large scales as compared to results in BPS17; see Section 4.6.

We recover a rather weak dependence on the $\mathrm{SC}$, but certain patterns can be discerned. The probability of recovering a bihelical, HSR-obeying spectrum is increased during the rising phase of the SC. Magnetic helicity tends to maximize not during the sunspot maximum but after some delay, and the descending phase is characterized with almost random kinds of helicity spectra. During the solar minimum, we observe an increased probability to find HSR-violating helicity spectra. These findings are in partial agreement with earlier work (sign change in between the ascending and descending phases), which has been reported before (e.g., Brandenburg et al. 2003) and for which also theoretical explanations have been proposed (e.g., Choudhuri et al. 2004; Pipin et al. 2013). Inexplicable features in our data (e.g., the reversed sign also at large scales, the highly variable behavior during the descending phase), however, remain.

One scenario that could explain the highly variable behavior in the descending phase are contributions arising from very complex ARs. In this work, we analyzed only one such region, but we were able to show that such a region can contribute significantly to the reversed helicity sign at intermediate scales. Their relative abundance to less complex ARs is known to be elevated during the descending phase of the SC (Jaeggli \& Norton 2016). Another possibility could be that signals from ARs occurring close to the equator might leak into the opposite hemisphere, thus polluting the spectrum from this hemisphere with the wrong sign. This scenario does not, however, explain the reversed signs of helicity at large scales.

By separating the magnetic fields associated predominantly with ARs into "weak" and "strong" fields, and determining the current helicity as well as the force-free parameter in these two regimes, Zhang (2006) found that the twistedness associated with only the weak magnetic fields obeys the sign rule. Thus, 
opposite signs of helicities were seen in weak- and strong-field regimes, giving evidence of a bihelical nature of magnetic fields. However, repeating the same procedure on a different data set resulted in an opposite finding where the strong fields were found to obey the sign rule while the weak fields carried the other sign of helicity (Gosain et al. 2013). Despite such different findings, it is interesting enough that the bihelicity could potentially be discerned in this way where the diffuse weak fields might represent the global/mean helicity while the strong fields could be more representative of the local/ fluctuating component carrying the opposite sign of the magnetic helicity.

As discussed in Section 4.4, the magnetic helicity spectra obtained during the early declining phase show intriguing features of clear HSR-obeying bihelicity, large power at the small wavenumbers, together with a Kazantsev spectral slope at large scales. It is not obvious whether systems with magnetic Prandtl number $\operatorname{Pr}_{\mathrm{M}}=\nu / \eta \ll 1$, with $\nu$ and $\eta$ being the kinematic viscosity and microscopic resistivity, respectively, must always host an SSD, which is much harder to excite in such a regime, making it somewhat an open issue whether the Sun, being a low-Pr ${ }_{M}$ object, indeed supports SSD. However, although dynamos at $\operatorname{Pr}_{\mathrm{M}}<1$ are harder to excite (Schekochihin et al. 2005), the adverse excitation conditions at $\operatorname{Pr}_{M}=0.1$ are now understood to be a consequence of the bottleneck effect in turbulence (Iskakov et al. 2007). This effect is particularly strong when turbulence is forced at the scale of the domain. Simulations of Subramanian \& Brandenburg (2014) at larger forcing wavenumbers resulted in no visible increase of the critical dynamo number. Once the dynamo is excited, the bottleneck effect is suppressed, so the low- $\operatorname{Pr}_{\mathrm{M}}$ controversy is hardly relevant in the nonlinear regime (Brandenburg 2014).

Based on the Kazantsev spectrum seen in Figure 7 and bearing in mind the discussion of the previous paragraph, we note that these results are suggestive of both LSD and SSD being operative simultaneously in the Sun. It remains to be seen how it all fits into a unified scheme of SSDs and LSDs such as the one explored by Subramanian (1999). More numerical works covering a sufficiently broad range of scales are needed in this interesting but complicated regime.

\section{Conclusions}

The present work has shown that the solar magnetic fields are bihelical, best observed during maximum activity of the Sun, with opposite signs of magnetic helicity at large and small length scales-exactly as expected from a helically driven global solar dynamo. Nearly $75 \%$ of all the analyzed synoptic maps show agreement with the HSR in terms of net magnetic helicity, which is dominated by ARs and thus becoming negative (positive) in the northern (southern) hemisphere. In agreement with some previous claims, the violations of the HSR are mostly seen during the early rising phase of the SC.

We have also highlighted the need for more reliable and better data, as it is possible that it is not the Sun but the data themselves that are more enigmatic, leading to opposite claims based on measurements from different instruments. We discussed one such example while noting some more from the literature. Therefore, improved data quality from upcoming missions such as Solar Orbiter with synergetic measurements from other facilities like DKIST is critical to establishing some fundamental claims about the solar helicity.
We thank the referee for useful suggestions and comments. This work utilizes SOLIS data obtained by the NSO Integrated Synoptic Program (NISP), managed by the National Solar Observatory, which is operated by the Association of Universities for Research in Astronomy (AURA), Inc., under a cooperative agreement with the National Science Foundation. Financial support from the Academy of Finland grant no. 272157 to the ReSoLVE Center of Excellence (M.J.K., P.J.K., and I.V.) and through the Max-Planck-Princeton Center for Plasma Physics (N.S.) is gratefully acknowledged. This research was further supported by the NSF Astronomy and Astrophysics Grants Program (grant 1615100) and the University of Colorado through its support of the George Ellery Hale visiting faculty appointment (A.B.).

\section{ORCID iDs}

Nishant K. Singh (iD https://orcid.org/0000-0001-6097-688X

Maarit J. Käpylä (i) https://orcid.org/0000-0002-9614-2200

Axel Brandenburg (iD https://orcid.org/0000-0002-7304-021X

Ilpo Virtanen (10 https://orcid.org/0000-0001-7258-4453

\section{References}

Arnold, V. I., \& Khesin, B. A. 1992, AnRFM, 24, 145

Balasubramaniam, K. S., \& Pevtsov, A. 2011, Proc. SPIE, 8148, 814809

Bao, S. D., Ai, G. X., \& Zhang, H. Q. 2000, JApA, 21, 303

Bao, S. D., Zhang, H. Q., Ai, G. X., \& Zhang, M. 1999, A\&A, 139, 311

Batchelor, G. K. 1953, The Theory of Homogeneous Turbulence (Cambridge: Cambridge Univ. Press)

Berger, M., \& Field, G. B. 1984, JFM, 147, 133

Bhat, P., Subramanian, K., \& Brandenburg, A. 2016, MNRAS, 461, 240

Blackman, E. G., \& Brandenburg, A. 2003, ApJ, 584, L99

Borrero, J. M., Lites, B. W., Lagg, A., Rezaei, R., \& Rempel, M. 2014, A\&A, 572, A54

Borrero, J. M., Tomczyk, S., Kubo, M., et al. 2011, SoPh, 273, 267

Bourdin, Ph.-A., Singh, N. K., \& Brandenburg, A. 2018, ApJ, submitted, (arXiv: 1804.04153)

Brandenburg, A. 2001, ApJ, 550, 824

Brandenburg, A. 2014, ApJ, 791, 12

Brandenburg, A., Blackman, E. G., \& Sarson, G. R. 2003, AdSpR, 32, 1835 Brandenburg, A., Petrie, G. J. D., \& Singh, N. K. 2017, ApJ, 836, 21 (BPS17) Brandenburg, A., \& Subramanian, K. 2005, PhR, 417, 1

Brandenburg, A., Subramanian, K., Balogh, A., \& Goldstein, M. L. 2011, ApJ, 734, 9

Charbonneau, P. 2010, LRSP, 7, 3

Chatterjee, P., Mitra, D., Brandenburg, A., \& Rheinhardt, M. 2011a, PhRvE, 84, 025403R

Chatterjee, P., Mitra, D., Rheinhardt, M., \& Brandenburg, A. 2011b, A\&A, 534, A46

Choudhuri, A. R., Chatterjee, P., \& Nandy, D. 2004, ApJL, 615, L57

Durrer, R., \& Caprini, C. 2003, JCAP, 0311, 010

Gosain, S., Pevtsov, A. A., Rudenko, G. V., \& Anfinogentov, S. A. 2013, ApJ, 772,52

Iskakov, A. B., Schekochihin, A. A., Cowley, S. C., McWilliams, J. C., \& Proctor, M. R. E. 2007, PhRvL, 98, 208501

Jaeggli, S. A., \& Norton, A. A. 2016, ApJL, 820, L11

Ji, H. 1999, PhRvL, 83, 3198

Kahniashvili, T., Tevzadze, A. G., Brandenburg, A., \& Neronov, A. 2013, PhRvD, 87, 083007

Käpylä, P. J., Käpylä, M. J., \& Brandenburg, A. 2009, A\&A, 500, 633

Käpylä, P. J., Mantere, M. J., \& Brandenburg, A. 2012, ApJL, 755, L22

Kazantsev, A. P. 1968, JETP, 26, 1031

Keller, C. U., Harvey, J. W., \& Giampapa, M. S. 2003, Proc. SPIE, 4853, 194

Krause, F., \& Rädler, K.-H. 1980, Mean-field Magnetohydrodynamics and Dynamo Theory (Oxford: Pergamon)

Lim, E.-K., Yurchyshyn, V., Park, S.-H., et al. 2016, ApJ, 817, 39

Liu, Y., Hoeksema, J. T., Bobra, M., et al. 2014a, ApJ, 785, 13

Liu, Y., Hoeksema, J. T., \& Sun, X. 2014b, ApJL, 783, L1

Longcope, D. W., \& Pevtsov, A. A. 2003, AdSpR, 32, 1845

Moffatt, H. K. 1969, JFM, 35, 117 
Moffatt, H. K. 1978, Magnetic Field Generation in Electrically Conducting Fluids (Cambridge: Cambridge Univ. Press)

Nindos, A., Zhang, J., \& Zhang, H. 2003, ApJ, 594, 1033

Ossendrijver, M. 2003, A\&A Rev., 11, 287

Ossendrijver, M., Stix, M., Brandenburg, A., \& Rüdiger, G. 2002, A\&A, 394, 735

Pariat, E., Leake, J. E., Valori, G., et al. 2017, A\&A, 601, A125

Pariat, E., Valori, G., Démoulin, P., \& Dalmasse, K. 2015, A\&A, 580, A128

Pevtsov, A. A., Berger, M. A., Nindos, A., Norton, A. A., \& van Driel-Gesztelyi, L. 2014, SSRv, 186, 285

Pevtsov, A. A., Canfield, R. C., \& Latushko, S. M. 2001, ApJL, 549, L261

Pevtsov, A. A., Canfield, R. C., \& Metcalf, T. R. 1995, ApJL, 440, L109

Pipin, V. V., \& Pevtsov, A. A. 2014, ApJ, 789, 21

Pipin, V. V., Zhang, H., Sokoloff, D. D., Kuzanyan, K. M., \& Gao, Y. 2013, MNRAS, 435, 2581

Roberts, P. H., \& Soward, A. M. 1975, AN, 296, 49

Rudenko, G. V., \& Anfinogentov, S. A. 2014, SoPh, 289, 1499

Schekochihin, A. A., Haugen, N. E. L., Brandenburg, A., et al. 2005, ApJL, 625, L115

Seehafer, N. 1990, SoPh, 125, 219
Seehafer, N. 1996, PhRvE, 53, 1283

Sheeley, N. R., Jr., \& Wang, Y.-M. 2015, ApJ, 809, 113

Solanki, S. K., Inhester, B., \& Schüssler, M. 2006, RPPh, 69, 563

Subramanian, K. 1999, PhRvL, 83, 2957

Subramanian, K., \& Brandenburg, A. 2014, MNRAS, 445, 2930

Thalmann, J., Pietarila, A., Sun, X., \& Wiegelmann, T. 2012, AJ, 144, 33

Trujillo Bueno, J., Shchukina, N., \& Asensio Ramos, A. 2004, Nature, 430, 326

Vainshtein, S. I., \& Zeldovich, Ya. B. 1972, SvPhU, 15, 159

Valori, G., Pariat, E., Anfinogentov, S., et al. 2016, SSRv, 201, 147

Warnecke, J., Brandenburg, A., \& Mitra, D. 2011, A\&A, 534, A11

Warnecke, J., Brandenburg, A., \& Mitra, D. 2012, JSWSC, 2, A11

Warnecke, J., Rheinhardt, M., Tuomisto, S., et al. 2018, A\&A, 609, A51

Yousef, T. A., \& Brandenburg, A. 2003, A\&A, 407, 7

Zhang, H., Brandenburg, A., \& Sokoloff, D. D. 2014, ApJL, 784, L45

Zhang, H., Brandenburg, A., \& Sokoloff, D. D. 2016, ApJ, 819, 146

Zhang, H., Moss, D., Kleeorin, N., et al. 2012, ApJ, 751, 47

Zhang, H., Sakurai, T., Pevtsov, A., et al. 2010, MNRAS, 402, L30

Zhang, M. 2006, ApJL, 646, L85 